\title{
Role of Simultaneous Source Technology in Seismic Industry
}

David McAdow ${ }^{*}$, , Jerry Krebs ${ }^{1}$, Partha Routh ${ }^{1}$, Anatoly Baumstein ${ }^{1}$, Sunwoong Lee ${ }^{1}$, Nathan Downey ${ }^{1}$, Chris Krohn ${ }^{1}$, Ramesh Neelamani ${ }^{2}$, and Dave Hinkley ${ }^{1},{ }^{1}$ Upstream Research Company, ExxonMobil, ${ }^{2}$ ExxonMobil Production and Development Company

Simultaneous source method has been investigated in the past in the context of efficient seismic data acquisition; however recently due to its computational efficiency in imaging, simulation and inversion the momentum in this field has increased. At ExxonMobil, simultaneous source technology has been an active research topic for many years in several areas ranging from acquisition, imaging and recently in full-wavefield inversion (FWI). More details can be obtained in the following externally published work:

- Simultaneous source in imaging (Jing et. al, 2001)

- Simultaneous source in reducing acquisition costs (Krohn et. al, 2006)

- Simultaneous source in reducing simulation cost in seismic wave propagation (Neelamani et. al, 2010)

- Simultaneous source in full-wavefield inversion (Krebs et. al, 2009; Baumstein et. al, 2011; Routh et. al, 2011)

With the increasing demand on better imaging capability in complex geological environments, the data volumes in the recent years are continually increasing. And to extract geophysical/geological information from these large volumes of data poses a significant computational challenge. Ultimately, this comes down to solving large scale seismic wave propagation problem efficiently. The key is in reducing the large number of individual shots down to one or a few encoded shots that provide significant computational savings. This computational savings impacts the forward and backward simulation in imaging problem (Jing et. al, 2001) and gradient computation in FWI. And if there is a mechanism to separate the simultaneous shots into individual shots it provides the ability to use this technology for faster simulation with large data sets (Neelamani et. al, 2010). Beyond its value in imaging and data acquisition we feel this technology has the potential to make significant impact in FWI related applications. In the time domain, the recent developments in encoded simultaneous source techniques for FWI have shown promise in significantly reducing the computational cost (Krebs et al., 2009; Baumstein et. al, 2011). We have further developed the simultaneous source inversion method that can handle moving receiver spreads such as marine streamer data (Routh et. al, 2011). In this talk we will present an overview of the research with simultaneous source technique at ExxonMobil and focus its value in FWI applications.

\section{References:}

- Baumstein, A., Ross, W., and Lee, S., 2011, Simultaneous source elastic inversion of surface waves, EAGE 2011

- Jing, X., Finn, C., Dicken, T., Willen, D., 2001, Encoding multiple shot gathers in prestack migration, SEG expanded abstracts, 19, 786-789.

- Krebs, J., Anderson, J., Hinkley, D., Neelamani, R., Lee, S., Baumstein, A., and Lacasse, M., 2009, Full-wavefield seismic inversion using encoded sources, Geophysics 74-6, WCC177-WCC188.

- Krohn, C. E., Johnson, M., Norris, M., and Ho, R., 2006, Vibroseis Productivity- Shake and go, SEG Expanded Abstracts, 42-46.

- Neelamani, R., Krohn, C., Krebs, J., Romberg, J., Deffenbaugh, M., and Anderson, J., 2010, Efficient seismic forward modeling using simultaneous random sources and sparsity, Geophysics, 75(6), WB15WB27

- Routh, P. Krebs, J., Lazaratos, S., Baumstein, A., Chikichev, I., Downey, N., Lee, S., Hinkley, D. and Anderson, J., 2011, Full-Wavefield Inversion of Marine Streamer Data with the Encoded Simultaneous Source Method, EAGE 2011 